\title{
New Porosimetric Method Based on the 3 $\gamma$ Annihilation Rate. Applications to Materials Science and Medical Imaging
}

\author{
B. JASIŃSKA* AND B. ZGARDZIŃSKA \\ Institute of Physics, M. Curie-Skłodowska University, pl. M. Curie-Skłodowskiej 1, 20-031 Lublin, Poland \\ A new parameter $\delta$ based on $3 \gamma$ annihilation is proposed. It describes the porosity of the materials containing \\ small intermolecular voids below $1 \mathrm{~nm}$. It can be applied to material investigation to compare changes in the \\ material porosity depending on temperature or pressure as well as to study of ageing or manufacturing processes. \\ Particularly it is dedicated to prepare the new imaging method and can be used during positron emission tomog- \\ raphy diagnosis allowing determination the kind or stage of pathogenic alteration.
}

DOI: 10.12693/APhysPolA.132.1616

PACS/topics: positron annihilation, positronium, $3 \gamma$ annihilation, porosity, positron emission tomography, medical imaging

\section{Introduction}

Techniques based on positron behaviour in the medium are widely used in material science for various properties investigation. The most common is probably the positron annihilation lifetime spectroscopy (PALS) as it has been applied to investigate free volume sizes. Hundreds of paper concerning investigation of various classes of porous materials was published. Positron and its bound state, positronium (Ps), became a widely used tool to follow interaction with matter.

Lifetime values of annihilating free positron and positronium provide information on the investigated matter local electron density. From free positron lifetime value the kind of defects in the conducting materials can be found while the ortho-positronium lifetime value brings information about free volume size in various classes of material, including polymers and porous media. The widely used Tao-Eldrup [1, 2] and extended Tao-Eldrup models [3] give possibility to investigate free volumes/pores in a wide radii range from about 0.1 to $50 \mathrm{~nm}[4,5]$.

The other technique based on positron behaviour in the dense matter is positron emission tomography (PET) [6], a diagnostic technique aiming three-dimensional imaging of the chosen substance metabolism in the patient body. Positron emitted from radiopharmaceutical annihilates with an electron from the human body. Two photons, $511 \mathrm{keV}$ each, are registered in two detectors creating line of response (LOR). From high number of registered events one can determine points of crossing and in effect places of much higher number of annihilation and, finally, a disease location. From the experience with the organic media investigation it is known that in the media like human tissues about $20-40 \%$ of positrons create positronium, however, this knowledge in PET diagnosis is completely neglected.

*corresponding author; e-mail: bozena.jasinska@umcs.pl
The aim of this paper is to propose a new indicator which can be applied to investigate material porosity in the case of the small (below $1 \mathrm{~nm}$ in size) free volumes. The proposed parameter can be applied both: in the material science to follow up changes in the porosity structure as well as to develop the new imaging method in PET, enabling the determination of changes in the tissue structure during tumour/cancer growth.

\section{Results and discussion}

\subsection{Definition of a new parameter $\delta$}

Positron-electron annihilation can undergo with the emission of $n$ photons $(\gamma)$ and the probability of annihilation is expressed as $1 /(372)^{n}$. It causes that almost all positrons annihilate emitting $2 \gamma$ while the probability of $3 \gamma$ process is less than $0.3 \%$. Both states of positronium (Ps) are unstable and annihilate with a mean lifetime value $125 \mathrm{ps}(p$-Ps) and $142 \mathrm{~ns}(o-\mathrm{Ps})$, respectively. In the vacuum the most probable way of $p$-Ps decay is with the emission of $2 \gamma$ while $o$-Ps decays with the emission of $3 \gamma$. In the dense matter, Ps during its lifetime can interact with the surroundings via many processes which influence both, the lifetime value and the annihilation probability. It is especially visible in the shortening of the $o$-Ps lifetime. In all kinds of media, where Ps creation is possible, the decay of $o$-Ps can undergo through two competitive processes: the intrinsic decay of triplet state and the pick-off process [7] - a bounded positron annihilates with an electron with an antiparallel spin orientation from the surroundings. The total $o$-Ps decay constant $\lambda_{o-P s}$ is then equal to

$$
\lambda_{o-P s}=1 / \tau_{o-P s}=1 / \tau_{p o}+1 / \tau_{t},
$$

where $\tau_{o-P s}$ is $o$-Ps lifetime value measured in the experiment, $\tau_{p o}$ is the lifetime value in the pick-off process, and $\tau_{t}$ is the lifetime value of the triplet state equal to $142 \mathrm{~ns}$. The longer is the $o$-Ps lifetime value, the larger is a fraction of $o$-Ps atoms decay via $3 \gamma, \mathrm{f}_{o-P s-3 \gamma}=\tau_{o-P s} / \tau_{t}$. The total $3 \gamma$ fraction is then a sum of small fraction from a free annihilation and the fractions from each o-Ps component 


$$
f_{3 \gamma}=\frac{1-\sum_{i} P_{i}}{372}+\frac{3}{4} \sum_{i} \frac{\tau_{i}(o-P s)}{\tau_{t}} P_{i} .
$$

The $i$ denotes the $i$-th $o$-Ps component, $P_{i}$ - the positronium formation probability of the $i$-th component. This expression is valid for the cases when the rate of $p$-Ps to $o$-Ps is equal to 1:3 which means that ortho-para spin conversion is not observed in the investigated material and positronium formation probability can be calculated from the intensity of respective components of the PAL spectrum.

The presented Eq. (2) can be applied to rough approximation of the $3 \gamma$ fraction only, as it is influenced by the extracted intensities of $o$-Ps components dependent on the efficiencies of detection of $2 \gamma$ and $3 \gamma$ events. However, it can be useful to compare $3 \gamma$ fraction in a group of investigated materials. The determination of the $3 \gamma$ fraction was widely applied to investigate high porosity materials towards preparation targets in some experiments in order to elongate positronium survival time and its production amount [8-10]. In a wide group of organic materials containing small intermolecular voids with the size below $1 \mathrm{~nm}$, determined $o$-Ps lifetime values are of an order of a few ns, while the intensity in most cases is in a range of $20-30 \%$. The expected $3 \gamma$ fraction will reach about $1 \%$. In order to emphasize the difference between the samples and make the comparison easier we propose to define a new parameter $\delta$ as a relative difference between $3 \gamma$ fraction in the investigated material (referred as $s$ ) and some reference material (ref):

$$
\delta_{3 \gamma}=\frac{\left(f_{3 \gamma}\right)_{s}-\left(f_{3 \gamma}\right)_{r e f}}{\left(f_{3 \gamma}\right)_{r e f}} \times 1000 \% \text {. }
$$

The defined parameter can be applied in material investigation, for example to observe changes in the porosity as a function of temperature or pressure and to follow modifications during material fabrication or ageing. The other possibility becomes useful during human body investigation with $\mathrm{PET}$ procedure as a morphometric indicator [11], the base of additional imaging method to the one used in current PET scanners based on the number of $2 \gamma$ annihilations.

\subsection{Applications to materials science}

Over the last few decades PALS was intensively used to investigate free volume fraction in polymers or pores structure in mesoporous materials. The lifetime value is commonly applied as a few existed models give a possibility to correlate the $o$-Ps lifetime value, determined from PALS, with the free volume/pore radii. The intensity is less applied as it is influenced by many processes undergoing in the material like presence of some ions, paramagnetic molecules or active groups [12]. In the simplest case, the $o$-Ps intensity is a function of free volumes concentration. A few proposals correlating the intensity and the number of the free volumes in polymers were published $[13,14]$. It was also proposed to use both $o$-Ps intensity and lifetime values to determine pores size distribution [15, 16]. Our proposal also includes these two parameters to follow investigated material porosity. The exemplary plot of $\delta$ parameter with the $o$-Ps lifetime and the intensity values as a function of temperature in octahedral silsesquioxanes $\mathrm{T}_{8}\left(\mathrm{CH}=\mathrm{CH}_{2}\right)_{8}$ is presented in Fig. 1. Discussion of PALS results were presented elsewhere [17]. Presented here the $\delta$ values were calculated in reference to value at the room temperature. In the $\delta$ value calculations, the third component was only taken into account. In the phase A both, lifetime and intensity, rise then calculated $\delta$ values also rise significantly. In the phase $\mathrm{B}$, it is difficult to conclude the porosity changes with temperature as the lifetime value increases and intensity decreases. The calculated $\delta$ values point out that below room temperature material porosity is almost constant (or slightly decreases) while above the room temperature it starts to increase slightly which is not visible from the lifetime and intensity dependences as a function of temperature. Perhaps it is worth looking for some additional process influencing material properties above room temperature.

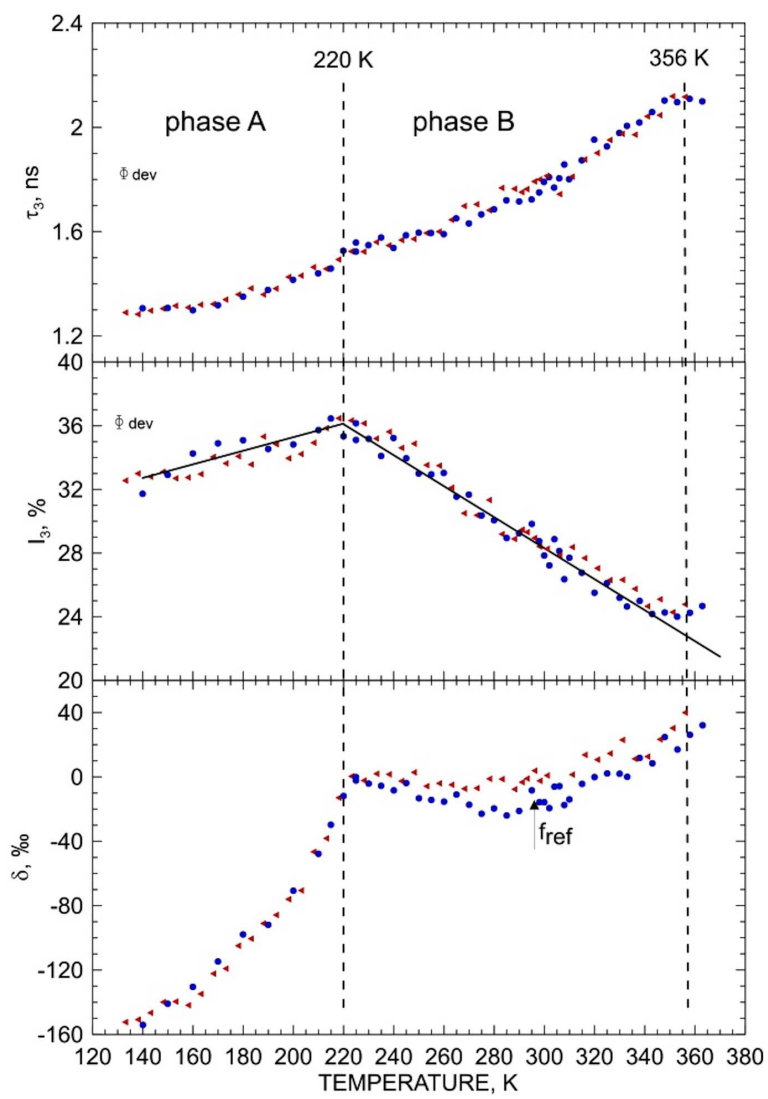

Fig. 1. Changes of $o$-Ps lifetime, intensity and $\delta$ parameter as a function of temperature in $\mathrm{T}_{8}\left(\mathrm{CH}=\mathrm{CH}_{2}\right)_{8}$ (down $\boldsymbol{\Lambda}$, up $\bullet$ ) Dashed lines - phase transition temperatures.

The next example of $\delta$ value application is presented in Fig. 2. The $\delta$ values were calculated for a group of long chain alkanes intensively investigated for the last few years [18-20]. From the data presented in the figure one can conclude that up to the phase transition the alkanes 


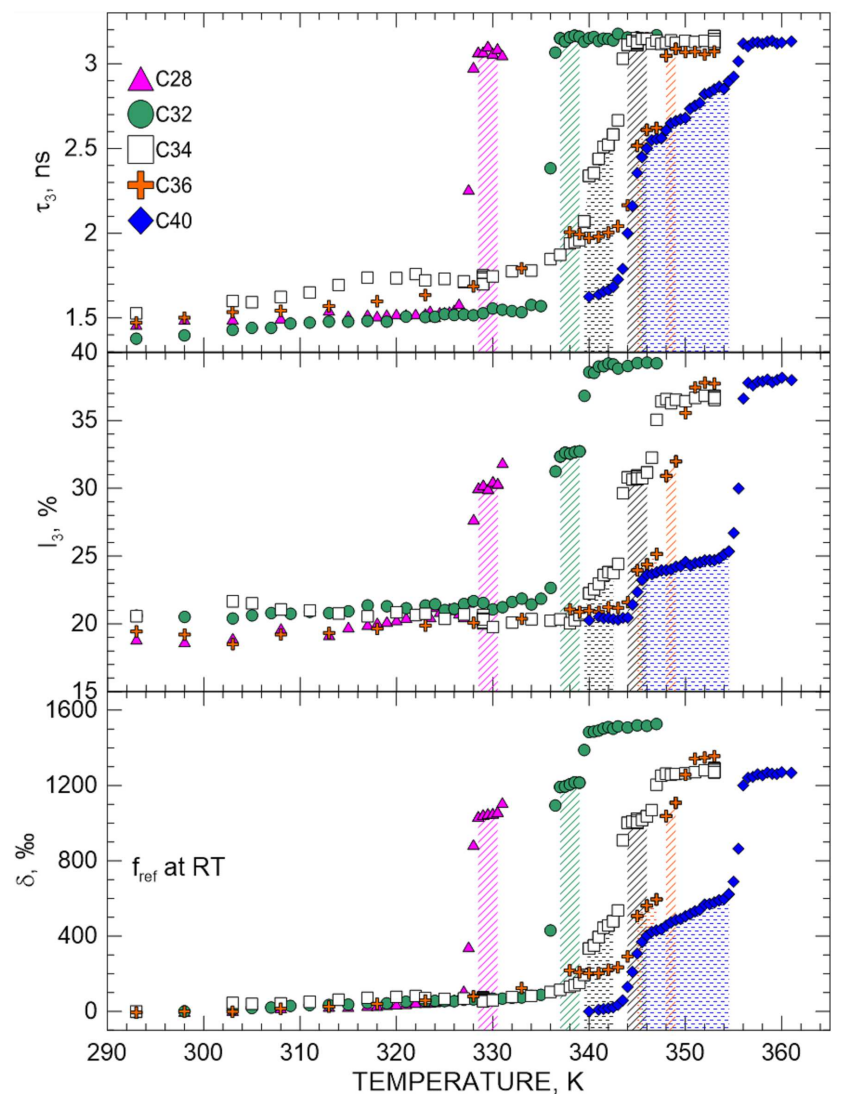

Fig. 2. The $o$-Ps lifetime, intensity and $\delta$ parameter as a function of temperature in selected long chain alkanes. Slashed area denotes rotator phase, dashed area — rigid monoclinic MI phase.

porosity, as defined by the $\delta$ indicator, is almost constant while in a rotator phase (dashed area) it rises more than twice. In rigid monoclinic MI phase it also rises but up to about $50 \%$ only.

\subsection{Applications to medical imaging}

The PET scanners, currently used in hospitals allow collecting two quanta produced during $2 \gamma$ positronelectron annihilation in coincidences. In order to apply the proposed $3 \gamma$ fraction to follow changes in material structure, the PET scanners should be equipped with the $3 \gamma$ coincidences system. The innovative tomography PET scanner allowing for the multi photon measurements is nowadays constructed by J-PET collaboration [21, 22]. It enables the diagnosis based on positron and positronium lifetime [23] as well as the one based on the ratio of $3 \gamma$ and $2 \gamma$ annihilation rates [24]. It is worth mentioning that using the lifetime value as a morphometric indicator in PET imaging of the most popular ${ }^{18} \mathrm{~F}$ radioisotope is not possible as this isotope decays directly to the ground state of its daughter nucleus. In the method based on $3 \gamma$ detection all kinds of radioisotopes can be applied.

The cancerous alteration could influence the tissues structure and in effect the free spaces size as well as the positronium formation probability connected to chemical processes in the cells. PALS techniques were applied successfully to investigate some living biological systems [25-28]. As it was mentioned above both factors: void sizes and concentration influence the $3 \gamma$ fraction. It is known that radiopharmaceutical concentration in the body depends on the metabolism rate in the tissues connected with cancerous alteration. That also causes changes in the number of produced $3 \gamma$ events. The ratio of three photon to two photon annihilation rates, $3 \gamma / 2 \gamma$, is concentration independent and will reflect only material changes. As a reference sample we propose using the PALS data for water, the main component of all biofluids in the living organism (Fig. 3).

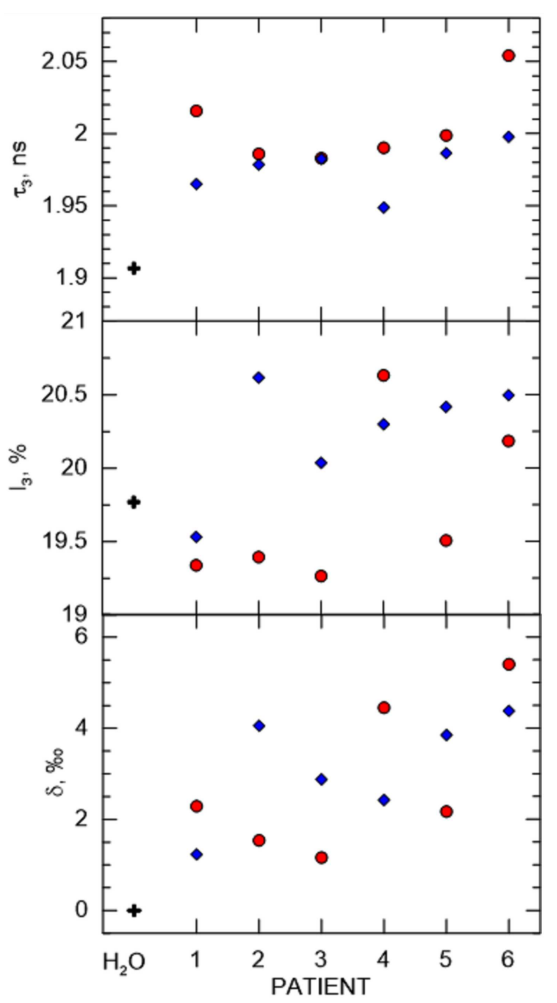

Fig. 3. The $o$-Ps lifetime, intensity and $\delta$ parameter for normal (blue diamonds) and altered (red circles) uterine tissues. Crosses denote values for deionized water at room temperature (reference point).

To verify the possibility of using the method based on positronium behaviour in the body in the PET investigations, a pilot study of human tissues was performed. Pairs of samples: normal (N) and altered (A) taken from patient uterus removed during a surgery were measured using PALS.

Figure 3 present the values of morphometric parameter $\delta$ calculated from Eq. (3). They are different from patient to patient but clear differences between healthy and tumorous tissues are also visible for each patient. These promising results give motivation to more detailed investigation and looking for correlation with other techniques. 


\section{Conclusions}

In the paper first attempt to apply $3 \gamma$ positron annihilation rate for porosity determination of the materials containing small voids below $1 \mathrm{~nm}$ is proposed.

The newly proposed porosity indicator based on $3 \gamma$ positron annihilation is defined by $o$-Ps lifetime and intensity as it can be simply calculated from PALS measurements but it can give additional informations to those commonly obtained from the PALS measurements. Actually it includes porosity rise coming from both: void radius (not volume) and concentration of the voids in the material structure. The $\delta$ indicator is a measure of a degree of the material porosity and its changes expressed in relation to some reference sample in order to simplify the results comparison.

The proposed indicator can be also applied to develop new imaging method in PET body examination. In this case when applied as a morphometric indicator it can serve for determination of the degree of tissue alteration. Preliminary investigations performed for pairs of normal and tumorous tissues indicate differences in both structures. One can expect that future investigations could help to recognize the stage of pathogenic changes or distinguish between different kinds of diseases of the same organ.

\section{References}

[1] S.J. Tao, J. Chem. Phys. 56, 5499 (1972).

[2] M. Eldrup, D. Lightbody, J.N. Sherwood, Chem. Phys. 63, 51 (1981).

[3] T. Goworek, K. Ciesielski, B. Jasińska, J. Wawryszczuk, Chem. Phys. 230, 305 (1998).

[4] B. Jasińska, A.L. Dawidowicz, Radiat. Phys. Chem. 68, 531 (2003).

[5] M. Śniegocka, B. Jasińska, R. Zaleski, T. Goworek, Chem. Phys. Lett. 430, 351 (2006).

[6] PET: Physics, Instrumentation and Scanners, Ed. M.E. Phelps, Springer, New York 2006.

[7] R.L. Garwin, Phys. Rev. 91, 1571 (1953).

[8] G. Consolati, R. Ferragut, A. Galarneau, F. Di Renzo, F. Quasso, Chem. Soc. Rev. 42, 821 (2013).

[9] R. Ferragut, S. Aghion, G. Tosi, G. Consolati, F. Quasso, M. Longhi, A. Galarneau, F. Di Renzo, J. Phys. Chem. C 117, 26703 (2013).

[10] R.S. Brusa, L. Di Noto, S. Mariazzi, G. Nebbia, J. Phys. Conf. Series 505, 012038 (2014).

[11] B. Jasinska, B. Zgardzińska, G. Chołubek, M. Gorgol, K. Wiktor, K. Wysogląd, P. Białas, C. Curceanu, E. Czerwiński, K. Dulski, A. Gajos, B. Głowacz, B. Hiesmayr, B. Jodłowska-Jędrych, D. Kamińska, G. Korcyl, P. Kowalski, T. Kozik, N. Krawczyk, W. Krzemień, E. Kubicz, M. Mohammed, M. PawlikNiedźwiecka, S. Niedźwiecki, M. Pałka, L. Raczyński, Z. Rudy, N. G. Sharma, S. Sharma, R. Shopa, M. Silarski, M. Skurzok, A. Wieczorek, H. Wiktor, W. Wiślicki, M. Zieliński, P. Moskal, Acta Phys. Pol. B 48, 1737 (2017); B. Jasińska, P. Moskal, Acta Phys. Pol. B 48, 1577 (2017).
[12] O.E. Mogensen, in: Positron Annihilation in Chemistry, Ed. V.I. Goldanskii, Springer-Verlag, Berlin 1995.

[13] Y. Kobayashi, W. Zheng, E.F. Meyer, J.D. McGervey, A.M. Jamieson, R. Simha, Macromolecules 22, 2302 (1989).

[14] G. Dlubek, G. Piontek, K. Ratzke, J. Kruse, F. Faupel, Macromolecules 41, 6125 (2008).

[15] R. Zaleski, J. Goworek, M. Maciejewska, Phys. Status Solidi C 6, 2445 (2009).

[16] R. Zaleski, W. Stefaniak, M. Maciejewska, J. Goworek, J. Coll. Interface Sci. 343, 134 (2010).

[17] A. Kowalewska, M. Nowacka, M. Włodarska, B. Zgardzińska, R. Zaleski, M. Oszajca, A. Krajenta, S. Kaźmierski, accepted to Phys. Chem. Chem. Phys., 2017. Manuscript ID: CP-ART-08-2017-005233.R1.

[18] T. Goworek, M. Pietrow, R. Zaleski, B. Zgardzińska, Chem. Phys. 355, 123 (2009).

[19] B. Zgardzińska, T. Goworek, Acta Phys. Pol. A 113, 1551 (2008).

[20] T. Goworek, J. Wawryszczuk, R. Zaleski, B. Zgardzińska, Radiat. Phys. Chem. 76, 185 (2007).

[21] A. Gajos, D. Kaminska, E. Czerwinski, D. Alfs, T. Bednarski, P. Białas, B. Głowacz, M. Gorgol, B. Jasińska, Ł. Kapłon, G. Korcyl, P. Kowalski, T. Kozik, W. Krzemień, E. Kubicz, M. Mohammed, Sz. Niedźwiecki, M. Pałka, M. Pawlik-Niedźwiecka, L. Raczyński, Z. Rudy, O. Rundel, N.G. Sharma, M. Silarski, A. Słomski, A. Strzelecki, A. Wieczorek, W. Wiślicki, B. Zgardzińska, M. Zieliński, P. Moskal, Nucl. Instrum. Methods Phys. Res. A 819, 54 (2016).

[22] D. Kamińska, A. Gajos, E. Czerwinski, D. Alfs, T. Bednarski, P. Białas, C. Curceanu, K. Dulski, B. Głowacz, N. Gupta-Sharma, M. Gorgol, B.C. Hiesmayr, B. Jasińska, G. Korcyl, P. Kowalski, W. Krzemień, N. Krawczyk, E. Kubicz, M. Mohammed, Sz. Niedźwiecki, M. Pawlik-Niedźwiecka, L. Raczyński, Z. Rudy, M. Silarski, A. Wieczorek, W. Wiślicki, B. Zgardzińska, M. Zieliński, P. Moskal, Eur. Phys. J. C 76, 445 (2016).

[23] P. Moskal et al., Patent Application: P 405185 (2013), PCT/EP2014/068374 (2014), WO2015028604.

[24] B. Jasińska, P. Moskal, Patent Application: P418689 (2016), PCT/IB2017/055560.

[25] Y.C. Jean, H.J. Ache, J. Am. Chem. Soc. 99, 1623 (1977).

[26] G. Liu, H. Chen, L. Chakka, J.E. Gadzia, Y.C. Jean, Phys. Status Solidi C 10, 3912 (2007).

[27] Y.C. Jean, Ying Li, Gaung Liu, Hongmin Chen, Junjie Zhang, J.E. Gadzia, Appl. Surf. Sci. 252, 3166 (2006).

[28] E. Axpe, T. Lopez-Euba, A. Castellanos-Rubio, D. Merida, J.A. Garcia, L. Plaza-Izurieta, N. Fernandez-Jimenez, F. Plazaola, J.R. Bilbao, PLoS One 2, 83838 (2014). 